\title{
Methods in Medicine
}

\section{Stabilisation of cleft maxilla using modified technique during bi-jaw orthognathic surgical procedures with simultaneous use of two stage surgical splints}

\author{
Maj S.S. Agarwal ${ }^{a, *}$, Brig B. Jayan ${ }^{b}$, Brig N.K. Sahoo ${ }^{c}$, Maj Amrit Thapa ${ }^{d}$ \\ ${ }^{a}$ Resident (Orthodontics), Dept of Dental Surg, Armed Forces Medical College, Pune 411040, India \\ ${ }^{\mathrm{b}}$ Consultant (Orthodontics), Army Dental Centre (R\&R), Delhi Cantt, India \\ c Professor \& Head, Dept of Dental Surg, Armed Forces Medical College, Pune 411040, India \\ ${ }^{\mathrm{d}}$ Graded Specialist (Orthodontics), Command Military Dental Centre (Central Command), Lucknow, India
}

\section{A R T I C L E I N F O}

Article history:

Received 25 June 2014

Accepted 1 August 2014

Available online 14 October 2014

Keywords:

Stabilisation of cleft maxilla

Bi-jaw orthognathic surgery

Surgery cleft maxilla

\section{Introduction}

Three dimensional stabilisation of maxilla in patients with orofacial clefts or craniofacial syndromes before, during and after bi-jaw orthognathic surgical procedures involving a large negative overjet is technically challenging to both the orthodontist and the maxillofacial surgeon. It is an important aspect of treatment planning and execution of bi-jaw surgical procedures involving cleft maxilla. During the surgery, maxilla should be stabilised with as rigid fixation as possible. ${ }^{1}$ Various techniques reported in the literature are by means of rigid plate fixation, intermaxillary fixation (IMF), tongue and groove locking osteotomies and occlusal splints etc. ${ }^{2}$ This paper highlights a novel approach for stabilisation of cleft maxilla during bi-jaw orthognathic surgical procedures which permits the concurrent use of intermediate and final splints during the surgical procedure.

\section{Material and methods}

A face bow is selected with the arch form and size approximating that of the patient's maxillary dentition with a passive

\footnotetext{
* Corresponding author. Tel.: +91 7507727658.

E-mail address: docshivagarwal@rediffmail.com (S.S. Agarwal).
} http://dx.doi.org/10.1016/j.mjafi.2014.08.003

0377-1237/@ 2014, Armed Forces Medical Services (AFMS). All rights reserved. 

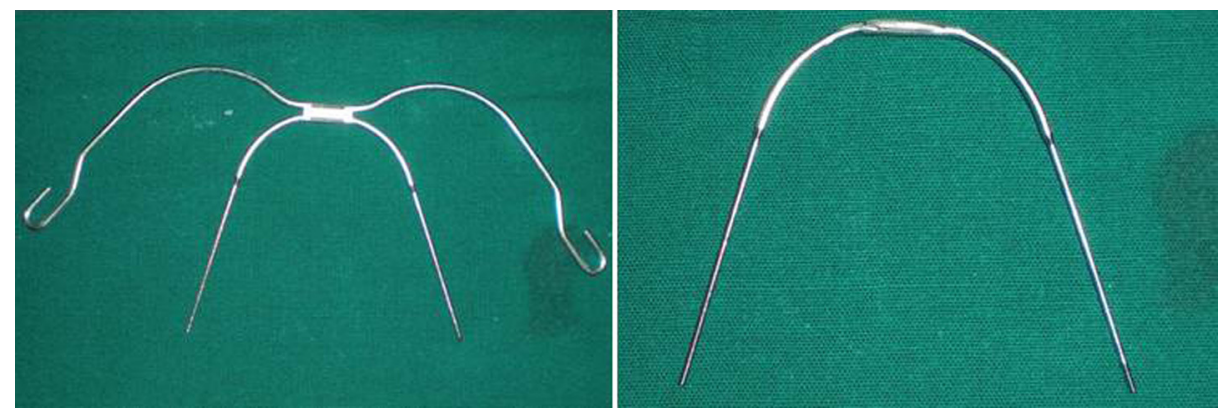

Fig. 1 - Inner bow of face bow.
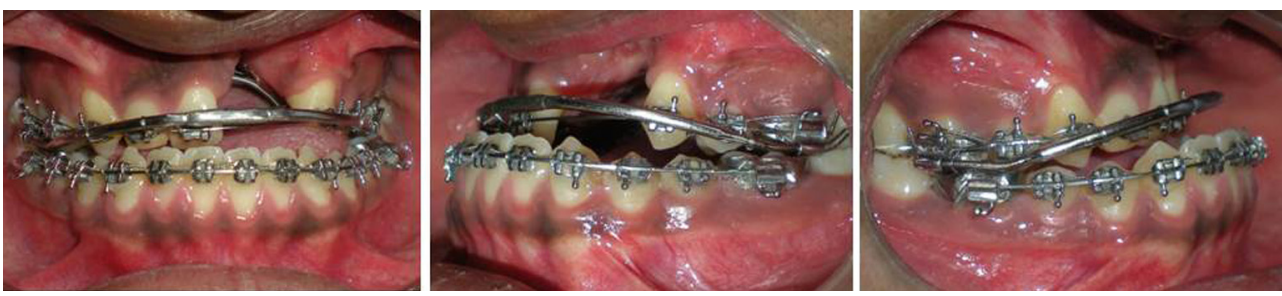

Fig. 2 - Modified face bow in situ.

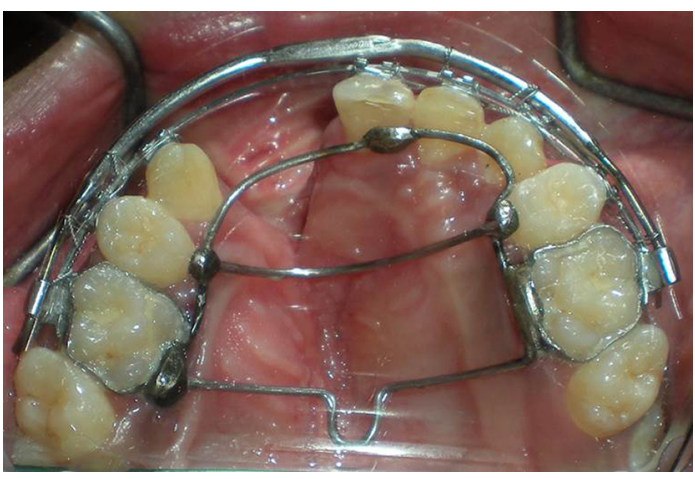

Fig. 3 - Modified palatal arch holding appliance.

fit. The outer bow is cut away and the joint between the inner and outer bow is rounded and polished (Fig. 1). Wax bite of the maxillary dentition is recorded and the inner bow is bent according to the arch extending about $1 \mathrm{~cm}$ distal to the molar tube of the last banded molar. Fit is checked by trial insertions and any necessary modifications are done. The distal ends on both sides are heat treated to enable bend back. A $45^{\circ}$ upward bend is given in the canine-premolar region bilaterally; firstly, to elevate the bow towards the vestibule so that it does not interfere with occlusion and secondly, to provide adequate access to the power arms of the molar tubes, premolar and canine brackets which would help in intermaxillary fixation during the surgery to secure the surgical splints in correct position and post surgically to put intermaxillary elastics (Fig. 2). A modified palatal arch holding appliance is placed in the maxilla prior to the placement of the inner bow to further enhance stability and unification of the cleft maxillary segments. It consists of a wire framework fabricated from $1 \mathrm{~mm}$ stainless steel wire which incorporates a transpalatal arch soldered to the molar bands with the wire extending anteriorly. Additional stability is provided by soldering a wire lingual to the first premolar on both sides (Fig. 3). The inner bow is then inserted into the headgear tubes of the molar tubes and cinched distally to resist dislodgement during intra and postoperative movements of the maxilla. The inner bow is then ligated to the palatal framework with the help of ligatures passing through the inter-dental region for unification. By this technique, the occlusal surfaces of the maxilla and mandible are left uncovered which will permit the use of intermediate splint (for establishing the planned position of maxilla during the first stage of bi-jaw surgery i.e. during the maxillary advancement) and final splint (for establishing the planned position of mandible during the second stage of bi-jaw surgery i.e. during mandibular setback). The intermediate splint is fabricated in pink and final splint is fabricated in clear acrylic
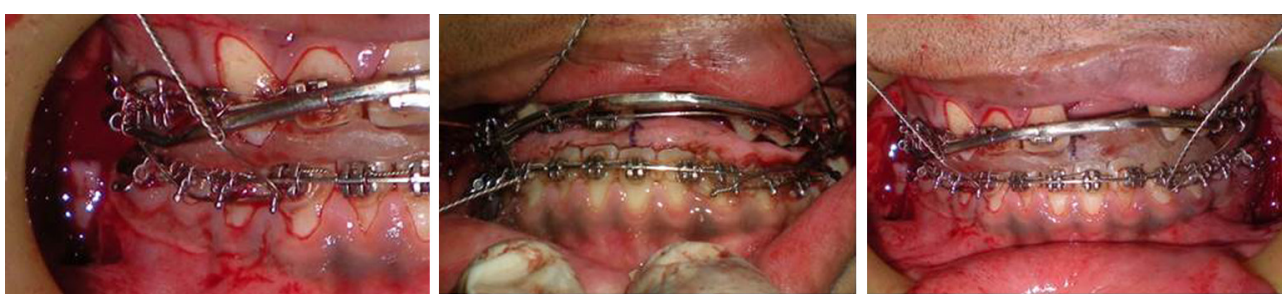

Fig. 4 - Intra operative stabilisation of intermediate (pink) and final splints (white). 
as a protocol to avoid confusion during surgery (Fig. 4). The inner bow is usually removed 2 weeks after surgery.

\section{Discussion}

Bi-jaw surgeries are generally indicated in patients with or without clefts with a negative overjet more than $10-12 \mathrm{~mm} \cdot{ }^{3,4}$ In our department, bi-jaw surgery is usually performed in cleft patients with a reverse overjet of greater then $10 \mathrm{~mm}$ and with compromised velopharyngeal mechanism. In such patients, bi-jaw surgery not only improves facial aesthetics but also has minimal negative influence on speech. The advantages of this technique are: securing of cleft maxilla with a rigid wire framework fixed palatally and buccally placed inner bow enhances rigidity, stabilises the maxilla as a single unit and facilitates use of intermediate and final splints which is not possible if cleft maxilla is stabilised with conventional methods like occlusal splint luted to the dentition. This technique also prevents intra operative and post-operative collapse of maxilla particularly in the transverse plane and hence, minimise post-treatment relapse. The limitations of this technique include: bilateral passive fit of the inner bow in the headgear tubes is required otherwise it may lead to undesired tooth movement; it cannot be used if the molars are not in acceptable alignment since bilateral passive fit of the inner bow is not possible in such situations and extra bulk in the vestibule may be uncomfortable to the patient.

This method can be used in cases treated with or without prior alveolar grafting. In cases where prior alveolar grafting has been done, the grafted area is generally weak and may not sustain the traction force used during the mobilisation of maxilla. So, in these cases the stabilising appliance adds to unify and stabilise the cleft maxillary segments during intraoperative procedures and post-operative take up of the graft. In non-grafted cases, simultaneous alveolar grafting is carried out along with the bi-jaw surgical procedure.
It should be noted that no instrument is used to mobilise the maxilla post down fracture in these patients as it may lead to dislodgement of the palatal framework. Bilaterally, maxillary posterior teeth are engaged with braided 26 gauge stainless steel wire and manually both the wires are pulled out, thereby advancing the maxilla.

\section{Conclusion}

This novel approach though routinely used for bi-jaw surgeries involving cleft maxilla, can also be used effectively in other situations like maxillary advancement or superior repositioning of maxilla in cleft patients.

\section{Conflicts of interest}

All authors have none to declare.

\section{R E F E R E N C E S}

1. Posnick J, Dagys A. Skeletal stability and relapse patterns after Le Fort I maxillary osteotomy fixed with miniplates: the unilateral cleft lip and palate deformity. Plast Reconstr Surg. 1994;94:924-932.

2. Burstein FD, Maurice SM, Granger M, Williams JK. Maxillary advancement in patients with severe maxillary hypoplasia: role of adjuvant stabilization techniques and materials. J Craniofac Surg. 2012;23(3):812-816.

3. Herber SC, Lehman JA. Orthognathic surgery in the cleft lip and palate patient. Clin Plast Surg. 1993;20:755-768.

4. Hirano A, Suzuki H. Factors related to relapse after Le Fort I maxillary advancement osteotomy in patients with cleft lip and palate. Cleft Palate Craniofac J. 2001;38(1):1-10. 